\title{
Entangled Mixed States and Local Purification
}

\author{
Adrian Kent \\ Department of Applied Mathematics and Theoretical Physics, University of Cambridge, \\ Silver Street, Cambridge CB3 9EW, U.K.
}

(28 May 1998; revised 23 August 1998)

\begin{abstract}
Linden, Massar and Popescu have recently given an optimization argument to show that a single two-qubit Werner state, or any other mixture of the maximally entangled Bell states, cannot be purified by local operations and classical communications. We generalise their result and give a simple explanation. In particular, we show that no purification scheme using local operations and classical communications can produce a pure singlet from any mixed state of two spin- $1 / 2$ particles. More generally, no such scheme can produce a maximally entangled state of any pair of finitedimensional systems from a generic mixed state. We also show that the Werner states belong to a large class of states whose fidelity cannot be increased by such a scheme.
\end{abstract}

The relationship between quantum entanglement and locality has been a source of great theoretical interest ever since the discovery of Bell's theorem, and new subtleties continue to be discovered. Several practical applications of entanglement have also been proposed, [1, 2, 5] most of which require that separated parties share a fixed maximally entangled state - conventionally, the Bell singlet state of two spin-1/2 particles. Since no preparation method is perfect and no transmission channel is noiseless, in practice we expect the parties to share entangled mixed states rather than pure singlets. This raises a problem which is also of independent theoretical interest: how can singlets be extracted from shared entangled mixed states?

One solution, at least in principle, is to use the "entanglement purification" scheme of Bennett et al. 四. But as this scheme relies on carrying out collective measurements on a large number of shared states, and produces perfectly pure singlets only when the number of shared states is infinite, it is natural to ask whether there is any simpler and more practical method. In particular, it is natural to ask whether there is any way of purifying individual entangled mixed states by a local purification scheme - that is, a scheme which involves only local measurements and unitary operations on the two entangled particles, together with classical communications between the parties.

Linden, Massar and Popescu (LMP) have recently addressed this question, showing that the answer is negative for the entangled Werner states [6] in two dimensions i.e., for rotationally invariant mixed states of two spin $1 / 2$ particles whose fidelity to the Bell singlet is greater than $1 / 2$. More generally, they show that the fidelity of a Werner state - i.e. the proportion of singlets cannot be increased by local purification schemes, and that no mixture of the maximally entangled Bell states of two spin-1/2 particles can be purified by such schemes to a state whose entanglement of formation is greater.
As LMP comment, these results may at first sight seem surprising, given that singlets can be produced from nonmaximally entangled pure states by individual measurements. 3] One might have conjectured that the same should be true of mixed states, at least if they are sufficiently close to being singlets. LMP's argument shows that this is not true, but does not give any clear intuition as to why pure and mixed states differ in this respect, and leaves open the possibility that generic mixed states of spin-1/2 particles, or mixed states of higher-dimensional systems, might be locally purifiable.

In this Letter we give a simple argument which generalises LMP's results and explains why individual mixed states cannot generally be completely purified. We show that no local purification scheme can produce a pure singlet from a single copy of any entangled mixed state of two spin 1/2 particles. More generally, we show that no local purification scheme can produce a maximally entangled state from a generic mixed state entangling any two finite-dimensional systems. Finally, we show that the Werner and Bell-diagonal states belong to a large class of states whose fidelity to a given state cannot be increased by any local purification scheme.

Consider first a general mixed state of two spin- $1 / 2$ particles,

$$
\rho=\sum_{i=1}^{n} p_{i}\left|\psi_{i}\right\rangle\left\langle\psi_{i}\right|
$$

where the $\left|\psi_{i}\right\rangle \in C^{2} \otimes C^{2}$ are distinct, the $p_{i}$ are positive, and $n \geq 2$ is minimal, i.e. $\rho$ cannot be represented as a mixture of fewer than $n$ pure states. Two parties, Alice and Bob, each have one of the spin- $1 / 2$ particles, and want if possible to carry out a sequence of local quantum operations and classical communications so as to obtain, with non-zero probability, the singlet

$$
\left|\Psi_{-}\right\rangle=\frac{1}{\sqrt{2}}(|\uparrow\rangle|\downarrow\rangle-|\downarrow\rangle|\uparrow\rangle)
$$

As the properties of a mixed state are independent of 
the details of its preparation we may, for clarity, suppose that a third party has actually prepared one of the pure states $\left|\psi_{i}\right\rangle$ for Alice and Bob, but gives them only the statistical information encoded in the decomposition (11) of $\rho$. In any local purification scheme, Alice and Bob carry out sequences of POV measurements, unitary rotations, and classical communications. They must either conclude from their measurement results that the purification has failed, in which case they jettison the state, or else - if the measurements lie in some specified subset of the possibilities - that it has succeeded. After any finite sequence of operations and measurements, the density matrix takes the form

$$
\rho^{\prime}=\frac{A \otimes B \rho A^{\dagger} \otimes B^{\dagger}}{\operatorname{Tr}\left(A \otimes B \rho A^{\dagger} \otimes B^{\dagger}\right)},
$$

where $A=A_{1} \ldots A_{n_{a}}$ and $B=B_{1} \ldots B_{n_{b}}$ are the products of the positive operators and unitary maps corresponding to Alice's and Bob's measurements and rotations.

Now if $\rho^{\prime}=\left|\Psi_{-}\right\rangle\left\langle\Psi_{-}\right|$then we must have that

$$
A \otimes B\left|\psi_{i}\right\rangle=a_{i}\left|\Psi_{-}\right\rangle,
$$

for each $i$ from 1 to $n$ and some constants $a_{i}$, which need not all necessarily be non-zero. But since $A \otimes B$ maps distinct rays onto the ray $\left|\Psi_{-}\right\rangle$, it cannot have maximal rank, which means that either $A$ or $B$ must have rank one or zero. This means that the $a_{i}$ must all be zero, contradicting the original hypothesis. The same argument holds true for an infinite sequence of local operations, so that no local purification scheme involving a countable number of steps can produce a singlet with non-zero probability.

Suppose now that Alice and Bob share a mixed state $\rho$ of two quantum systems described by spaces $H_{A}$ and $H_{B}$ of dimensions $N_{A} \geq 2$ and $N_{B} \geq 2$. We can write

$$
\rho=\sum_{i=1}^{r} p_{i}\left|\psi_{i}\right\rangle\left\langle\psi_{i}\right|
$$

where the $\left|\psi_{i}\right\rangle$ are orthogonal, the $p_{i}$ are positive, and $r$ is the rank of $\rho$. Any sequence of local operations can be implemented by an operator $A \otimes B$ as in (3), and if these operations purify $\rho$ to some state $|\Psi\rangle$ then we have $A \otimes B\left|\psi_{i}\right\rangle=a_{i}|\Psi\rangle$ for each $i$ from 1 to $r$ and some constants $a_{i}$, so that the rank of $A \otimes B$ is at most $N_{A} N_{B}-r+1$. If $r \geq N_{A} N_{B}-2$, as is true for generic $\rho$, this implies that at least one of $A$ and $B$ has rank one or zero, so that $|\Psi\rangle$ cannot be entangled. Hence no local purification scheme can produce an entangled pure state from a generic mixed state of two separated finitedimensional systems.

Since the set of possible purification operators $A \otimes B$ is compact, any state which cannot be completely purified to a maximally entangled state must have a maximal purifiability - according to any continuous measure - whenever all the purification operators produce a welldefined state. Moreover, there must be some local purification for which this maximum is attained. For example, a generic mixed state $\rho$ of two finite-dimensional systems has maximal rank:

$$
\rho=\sum_{i=1}^{N_{A} N_{B}} p_{i}\left|\psi_{i}\right\rangle\left\langle\psi_{i}\right|,
$$

where the $\left|\psi_{i}\right\rangle$ are orthonormal. Thus $\operatorname{Tr}\left(A \otimes B \rho A^{\dagger} \otimes B^{\dagger}\right)$ is zero only if $A \otimes B$ is the zero operator. Since without loss of generality we can take $\|A\|=\|B\|=1$, it follows that there is some $\epsilon(\rho)>0$ such that

$$
\max _{A, B ;|\psi\rangle} \frac{\left\langle\psi\left|A \otimes B \rho A^{\dagger} \otimes B^{\dagger}\right| \psi\right\rangle}{\operatorname{Tr}\left(A \otimes B \rho A^{\dagger} \otimes B^{\dagger}\right)}=1-\epsilon(\rho),
$$

where the maximum is taken over all local purification operators $A$ and $B$ and all maximally entangled pure states $|\psi\rangle$.

It is perhaps worth stressing that this does not contradict the results of Bennett et al., [4] whose scheme produces a non-zero fractional yield of pure Bell singlets only in the asymptotic limit as the number of purified pairs tends to infinity. Nor does the result apply to all mixed states: it is easy to construct examples of mixed states of lower rank which can be purified to singlets. Examples of lower rank mixed states which can be purified to arbitrary high fidelity, though not fidelity 1, are also known. [8]

Finally, we consider the problem of whether the proportion of some entangled pure state in a mixture can be increased by a local purification scheme. Suppose that

$$
\rho_{F}=F|\Psi\rangle\langle\Psi|+(1-F) \tilde{\rho}
$$

is a density matrix, where $|\Psi\rangle$ is a normalised entangled state, $0 \leq F \leq 1$, and $\tilde{\rho}$ is a density matrix with $\langle\Psi|\tilde{\rho}| \Psi\rangle=0$. Suppose also that there is some $F_{0}$ with $0<F_{0}<F$ such that $\rho_{F_{0}}$ is not entangled, i.e. $\rho_{F_{0}}$ can be written as a convex combination of non-entangled pure states, and such that any state with fidelity greater than $F_{0}$ to $|\Psi\rangle$ is entangled.

Then we can show that no local purification scheme can produce a mixed state $\rho^{\prime}$ from $\rho_{F}$ such that the fidelity $F^{\prime}=\left\langle\Psi\left|\rho^{\prime}\right| \Psi\right\rangle$ of $\rho^{\prime}$ to the state $|\Psi\rangle$ is greater than $F$. As before, we can describe the action of any successful local purification scheme by (3). If we consider the scheme acting on $\rho_{F}$, it produces a state of fidelity

$$
\begin{aligned}
& F^{\prime}(F)= \\
& \quad \frac{F|\langle\Psi|A \otimes B| \Psi\rangle|^{2}+(1-F)\left\langle\Psi\left|A \otimes B \tilde{\rho} A^{\dagger} \otimes B^{\dagger}\right| \Psi\right\rangle}{\operatorname{Tr}\left(A \otimes B(F|\Psi\rangle\langle\Psi|+(1-F) \tilde{\rho}) A^{\dagger} \otimes B^{\dagger}\right)},
\end{aligned}
$$

with probability

$$
\operatorname{Tr}\left(A \otimes B(F|\Psi\rangle\langle\Psi|+(1-F) \tilde{\rho}) A^{\dagger} \otimes B^{\dagger}\right) .
$$


Note that if this probability is non-zero for any value of $F$ in the range $0<F<1$ then it is non-zero throughout the range.

We have that

$$
\begin{aligned}
& \frac{d^{2}}{d F^{2}} \delta(F)= \\
& \quad \frac{\beta^{\prime}}{\left(\operatorname{Tr}\left(A \otimes B(F|\Psi\rangle\langle\Psi|+(1-F) \tilde{\rho}) A^{\dagger} \otimes B^{\dagger}\right)\right)^{3}},
\end{aligned}
$$

where $\delta(F)=\left(F^{\prime}(F)-F\right)$ and $\beta^{\prime}$ is independent of $F$, so that the sign of the second derivative is constant on the range $0<F<1$ and $\delta(F)$ is either convex, concave or linear over the range. Now $\delta(F) \geq-F$ and so approaches or exceeds zero as $F \rightarrow 0$ from above; similarly $\delta(F)$ approaches or is less than zero as $F \rightarrow 1$ from below. By hypothesis $\delta\left(F_{0}\right) \leq 0$, since it is impossible to obtain an entangled state from the unentangled state $\rho_{F_{0}}$. Hence, if there is an $F$ in the range $F_{0}<F<1$ such that $\delta(F)>0$, then, given the form of $(9), \delta(F)$ must have at least two extrema in the range $0<F<1$, which is inconsistent with (11). So there can be no such $F$. Hence no local purification scheme can increase the fidelity of $\rho_{F}$ for $F \geq F_{0}$.

As a special case, applying the result to a pair of spin$1 / 2$ particles, taking $|\Psi\rangle$ to be the Bell singlet state $\left|\Psi_{-}\right\rangle$and $\tilde{\rho}$ to be a linear combination $a\left|\Psi_{+}\right\rangle\left\langle\Psi_{+}\right|+$ $b\left|\Phi_{-}\right\rangle\left\langle\Phi_{-}|+c| \Phi_{+}\right\rangle\left\langle\Phi_{+}\right|$, we obtain LMP's result that the fidelity of a Werner state, or any Bell-diagonal state, with $F>\frac{1}{2}$ cannot be increased by a local purification scheme.

In summary, the difficulty with purifying individual mixed states is that any purification scheme must have a well-defined action on each of the pure states in the mixture, and hence on all other mixtures of those states, including any non-entangled mixtures. This imposes strong constraints, which do not arise in purifying pure states, and which make effective purification of mixed states generally inconsistent with locality. We have used only the very simplest constraints here in generalising LMP's results: more detailed analyses would no doubt give stronger results and bounds.

These results remove any remaining hope that noise on a quantum channel can be completely countered by individual measurements on mixed states, whether or not the noise is rotationally symmetric. As LMP's results already strongly suggest, if teleportation and similar schemes are to become workable, they will require either essentially noisefree channels, or algorithms tolerant to noise, or technology that allows efficient collective measurements.

Acknowledgements I am very grateful to Michal Horodecki, Noah Linden, Serge Massar, Martin Plenio and Sandu Popescu for helpful discussions and to the Royal Society for financial support.

[1] C. Bennett et al., Phys. Rev. Lett. 70 (1993) 1895

[2] A. Ekert, Phys. Rev. Lett. 68 (1991) 661

[3] C. Bennett, H. Bernstein, S. Popescu and B. Schumacher, Phys. Rev. A 53 (1996) 2046

[4] C. Bennett et al., Phys. Rev. Lett. 76 (1996) 722

[5] D. Deutsch et al., Phys. Rev. Lett. 77 (1997) 2818; erratum, Phys. Rev. Lett. 80 (1998) 2022.

[6] R. Werner, Phys. Rev. A 40 (1989) 4277

[7] N. Linden, S. Massar and S. Popescu, quant-ph/9805001

[8] M. Horodecki, P. Horodecki and R. Horodecki, quant$\mathrm{ph} / 9807091$ 